\title{
Modeling The Population of File-Sharing Peer-to-Peer Networks With Branching Processes
}

\author{
Trang Dinh Dang, Roland Pereczes, Sándor Molnár \\ High Speed Networks Laboratory, Dept. of Telecommunications and Media Informatics \\ Budapest Univ. of Technology and Economics, H-1117, Magyar Tudósok krt. 2., Budapest, Hungary \\ Tel: (361) 463 3664, Fax: (361) 463 3107, E-mail: \{trang,molnar\} @ tmit.bme.hu
}

\begin{abstract}
In this paper we develop a simple but effective mathematical model to capture the file population dynamics of file-sharing peer-to-peer systems. Our modeling framework is based on the theory of branching processes. We describe analytically the behavior of the proposed model. The precise characterization of the necessary and sufficient conditions of population extinction or explosion is given based on the system parameters. We also present the expected ratio of active, passive and dead peers for the long-term regime. We validate and demonstrate our results in several simulation studies. Based on our results we propose a number of engineering guidelines to the design and control of file-sharing P2P systems.
\end{abstract}

\section{INTRODUCTION}

Recent traffic measurements (e.g. [1]) show that the workload generated by P2P applications are the dominant part of most of the Internet segments. In spite of the fact that the popularity of current P2P applications changes fast, it seems that the file sharing-like applications were, are and probably will be the most popular application type among all the P2P applications. P2P file sharing also shows an evolution starting from Napster and going through many new developments resulted in Gnutella, Kazaa, Morpheus, eDonkey, BitTorrent, etc.

In this paper we analyze the population dynamics of a filesharing peer-to-peer system. We build up a general model which is capable of capturing all the important characteristics of relevant P2P file-sharing systems. We perform a comprehensive performance analysis based on the theory of branching processes. We investigate the characteristics of the system and present several results about the necessary and/or sufficient conditions of extinction, stagnation and explosion of the population size of shared files. Our analytical results are validated by a simulation study and we also present a number of examples about the evolution of population size in different cases. Finally, we derive a number of useful engineering guidelines from the results which may help the design and the control of peer-to-peer file sharing systems.

\section{A. Related work}

Most of the early P2P research was mainly focused on traffic measurements and design. These fields are still active and recently several studies were published reporting results on these areas with related characterization studies, e.g. [9]. On the other hand, the performance evaluation of P2P systems is becoming a hot topic of recent research. Starting from
[10] where a closed queueing system is used to analyze the performance of a P2P system a number of new results were published trying to get some more understanding about the behavior of such systems. Focusing only on the topic of this paper papers [2], [3], [4], [5], [6], [7], [8] are the most closely related published results.

In [5] the authors studied the service capacity of a P2P system both in the transient regime with a branching process model and also in the stationary regime with a Markov chain model. They have found, among others, an exponential growth of service capacity during the transient phase. Several papers focused on the currently popular BitTorrent P2P applications, e.g. [2], [8], [4], [7]. The authors of [2] have applied a fluid model to reveal the performance and scalability aspects of BitTorrent. [8] presents an extensive trace analysis and modeling study of BitTorrent-like systems. The recent paper [4] uses a deterministic fluid model and a Markov chain to study the system behavior and an approximation for the life time of a chunk in BitTorrent is also proposed. The behavior of the peers in BitTorrent is studied in the recent paper [7], where the authors also investigate the file availability and the dyingout process. The population dynamics of the P2P systems is also addressed in [3], where a spatio-temporal model is proposed to analyze the resource usage of the system.

The main difference between these papers and our paper is that our analysis is entirely based on the theory of branching processes. We create a reasonable model for filesharing P2P system and derive a detailed characterization of the system in a particular way. In the most related previous work the authors in [5] also applied a branching process model but their analysis was restricted to showing the sensitivity of the exponential growth behavior to the system parameters in the transient regime. [5] uses the simplest branching model to explain the basis of population growing without any consideration of a real P2P system operation.

\section{Modeling File Population of P2P Systems}

The objective of the proposed model is to describe the main characteristics of P2P file-sharing systems: the population of shared files. Technically, all the available $\mathrm{P} 2 \mathrm{P}$ file-sharing systems apply the same rule. P2P users contribute to the common system resource by providing the access to a set of their files and they have access to the common resource in return. In general, the common resource consists of one 


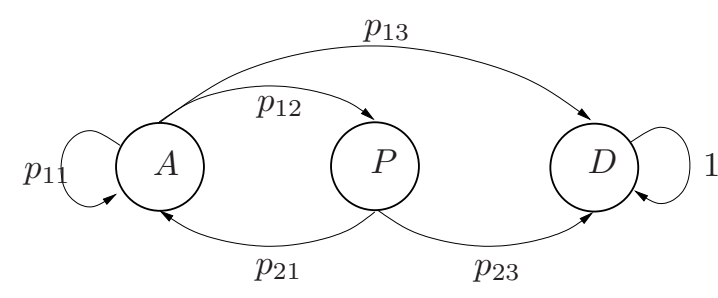

Fig. 2. The state transitions and probabilities

have little impact on the overall population of the system in general. Therefore we assume only single and complete parent model in our description. In other words, a file can be shared only if it is complete and it has the origin from only one peer.

Combining these characteristics we propose a model of agedependent multitype branching process for $\mathrm{P} 2 \mathrm{P}$ file-sharing system. We differentiate between two types of peers owning the concerned file: cooperating peers and free riders. After the successful download of a file the cooperating peers will share the file with the system, contributing to the newer copies of the file in the system. Further, cooperating peers have two possible states, active (A) and passive (P), corresponding to their online and offline activities. An online peer can give rise to a new copy while offline peers are unaccessible, thus do not create new offsprings. Non-cooperating peers or free riders are considered as dead (D) peers (copies), since they do not contribute to the birth of any offsprings. The possible transitions between states and the corresponding probabilities are shown in Fig. 2.

We assume that an active peer can only change its state when the offspring is born. To be more specific, when an individual is born, it has to choose to be active, passive or non-cooperative, i.e. 'dead'. If the individual is active, it will stay in this state until its offspring is born. State transitions of active peers only happen at these instants.

In the model we use the following assumptions and notations:

- The age time, i.e. the age of the parent when offspring is born, is a random variable $T_{a}$ with an exponential distribution with mean $\alpha$. A peer can have several offsprings during their activity time (lifetime) in the system. The age of the parent is counted from the parent's activation time, i.e. when it turns from passive to active or it is just born, or from the birth of the last born offspring (see Fig. 1).

- The offline time of a peer (length of passive period) is also an exponential variable $T_{p}$ with mean $\beta$.

- The expected value of offsprings in a single birth is $\lambda$. This parameter expresses the average number of exact parallel downloads from a peer. In the P2P case $\lambda \equiv 1$, since the probability that multiple downloads of a file from a peer end at the same time is zero. However, we use $\lambda$ in the general discussion of the model.

- Let $\left\{\pi_{i}\right\}_{i=1}^{3}$ be the probabilities that a new peer becomes active, passive or dead. Clearly, $\sum_{i=1}^{3} \pi_{i}=1$. From this point the lower indices 1,2,3 will refer to active, passive and dead peer states.
- The type-transition matrix which describes the probabilities of state transitions is the following:

$\begin{gathered}A \\ A \\ P \\ D\end{gathered} \quad\left[\begin{array}{ccc}p_{11} & p_{12} & p_{13} \\ p_{21} & 0 & p_{23} \\ 0 & 0 & 1\end{array}\right]$,

where $A, P$ and $D$ stand for active, passive and dead state, respectively. For example: $\mathbf{P}[$ active $\rightarrow$ dead $]=p_{13}$. Obviously, $\sum_{j=1}^{3} p_{1 j}=1, p_{21}+p_{23}=1$ since the typetransition matrix is a stochastic matrix.

With the assumptions of the memoryless property of the age time $T_{a}$ and the offline time $T_{p}$ the population size process is Markovian. The next section derives the expected size of the population and the most important features of the process.

\section{ANALYSIS OF THE MODEL}

\section{A. Model description}

In this subsection we show how our branching process model can be characterized by its transition operator and we derive the operator parameters from our P2P system model parameters.

Theoretically, if the generating function [1] of a branching process is known, then all important properties of the process is determined (e.g. the extinction probability, the expected population size, the deviation of size). Unfortunately, to determine the generating function of the proposed branching model, the following probabilities should be calculated:

$\mathbf{P}$ [the number of active and passive peers at time $t_{0}$ is $\left.(k, l)\right]$, which is a very complicated task. Therefore we avoid the use of generating functions.

Let $Z_{t}=\left(Z_{t}^{(1)}, Z_{t}^{(2)}, Z_{t}^{(3)}\right)$ be the vector representing the population size of active, passive and dead peer at the time instant $t$. Let $M_{t}$ be the transition operator defined by:

$$
Z_{t}=M_{t} Z_{0} .
$$

It is easy to see that the process we investigate depends linearly on $Z_{0}$, which means that $M_{t}$ is a random matrix.

First of all, since the process is Markovian it can be realized that the following equation holds:

$$
M_{t+s}=M_{t} M_{s}
$$

where $M_{t}$ and $M_{s}$ are independent random matrices. This implies:

$$
\lim _{n \rightarrow \infty}\left(\mathbf{E} M_{t / n}\right)^{n}=\mathbf{E} M_{t}
$$

The element $\left(\mathbf{E} M_{t}\right)_{1,1}$ of the matrix $\mathbf{E} M_{t}$ can be determined as follows. Let us choose an appropriate small time interval $\delta \in \mathbb{R}^{+}$, such that the probability that two or more downloads are finished in $\delta$ is $o(\delta)$, where $\lim _{\delta \rightarrow 0} \frac{o(\delta)}{\delta}=0$. Thus the probability that an active peer is going to have children within the time interval $\delta$ is $\frac{\delta}{\alpha}+o(\delta)$. Similarly, we have:

$\mathbf{P}[$ a passive peer becomes active within $\delta]=\frac{\delta}{\beta}+o(\delta)$. 
The average number of active peers produced by one active peer after the time interval $\delta$ is $\lambda \pi_{1} \frac{\delta}{\alpha}$ because there are $\lambda$ offsprings on average and only $\lambda \pi_{1}$ will be active. But an active peer may also become passive or dead with probabilities $p_{12}$ and $p_{13}$; the probability that no file-sharing happens is $1-\frac{\delta}{\alpha}+o(\delta)$. Then,

$$
\left(\mathbf{E} M_{\delta}\right)_{1,1}=\frac{\delta}{\alpha} \lambda \pi_{1}+\left(1-p_{12}-p_{13}\right) \frac{\delta}{\alpha}+\left(1-\frac{\delta}{\alpha}\right) .
$$

Using similar considerations $\mathbf{E} M_{\delta}$ is given by:

$$
\mathbf{E} M_{\delta}=\underbrace{\left[\begin{array}{lll}
1 & 0 & 0 \\
0 & 1 & 0 \\
0 & 0 & 1
\end{array}\right]}_{I}+\delta \underbrace{\left[\begin{array}{lll}
\frac{\lambda \pi_{1}+p_{11}-1}{\alpha} & \frac{p_{21}}{\beta} & 0 \\
\frac{\lambda \pi_{2}+p_{12}}{\alpha} & -\frac{1}{\beta} & 0 \\
\frac{\lambda \pi_{3}+p_{13}}{\alpha} & \frac{p_{23}}{\beta} & 0
\end{array}\right]}_{A}+o(\delta) .
$$

Letting $\delta=\frac{t}{n}$, we get

$\mathbf{E} M_{t}=\lim _{n \rightarrow \infty}\left(\mathbf{E} M_{t / n}\right)^{n}=\lim _{n \rightarrow \infty}\left(I+\frac{A t}{n}\right)^{n}=\exp (A t)$

(7) implies that $\mathbf{E} Z_{t}$ grows exponentially with a rate determined by the eigenvalues of $A$. Let $\gamma_{i}, i=1,2,3$ be the eigenvalues of $A$. It is clear that one of them is zero. Put $\gamma_{3}=0$. The other two eigenvalues of $A$ are given by:

$$
\gamma_{1,2}=\frac{-b \pm \sqrt{b^{2}-4 c}}{2}
$$

where

$b:=-\left(a_{22}+a_{11}\right)=\frac{1}{\beta}-\frac{\lambda \pi_{1}+p_{11}-1}{\alpha}$,

$c:=a_{11} a_{22}-a_{12} a_{21}=\frac{\left(1-\lambda \pi_{1}-p_{11}\right)-p_{21}\left(\lambda \pi_{2}+p_{12}\right)}{\alpha \beta}$,

$a_{i j}$ is the $(i, j)$-th element of $A$.

Considering (8) one can clearly see that $\gamma_{1,2}$ are real numbers since $a_{12}, a_{21} \geq 0$. In addition, $\gamma_{1}=\gamma_{2}$ if and only if

$$
\left\{\begin{array}{l}
a_{11}=a_{22}=-\frac{1}{\beta}<0 \\
p_{21}=0 \text { or }\left(\pi_{2}=0 \text { and } p_{12}=0\right)
\end{array},\right.
$$

i.e., $\gamma_{1}=\gamma_{2}<0$ and either $p_{12}=0$ or $p_{21}=0$. This means that either active peers cannot become passive or passive ones cannot become active. This is completely unlikely and unrealistic regarding the concerned systems. Thus the investigation of this case is ignored. Put $\gamma_{1}>\gamma_{2}$.

\section{B. The expected population size of the process}

Using the model description presented above some important properties of the system can be derived. In this subsection we present several necessary and/or sufficient conditions of extinction, stagnation and explosion of the population size of shared files. The ratio of active, passive and dead peers in the long-term behavior is also provided.

Since the maximal eigenvalue of $A$ determines the behavior of the process, it is worth differentiating between two cases:

- $\max \left\{\gamma_{i}\right\}>0$, i.e. there exists at least a positive eigenvalue of $A$ (so $\left.\gamma_{1}>0\right)$

- $\max \left\{\gamma_{i}\right\}=0$, i.e. $\gamma_{1,2} \leq 0$.
Lemma 1: If the condition $\left(\frac{\pi_{1}}{\pi_{2}} \leq 1+\frac{p_{13}}{p_{12}}\right)$ holds, the matrix $A$ has positive eigenvalue(s) if and only if

$$
\exists i: \gamma_{i}>0 \quad \Leftrightarrow \quad \lambda>\frac{\left(p_{12}+p_{13}\right)+p_{12} p_{21}}{\pi_{1}+\pi_{2} p_{21}}
$$

Note that $\lambda=1$ in our particular model.

The next statement provides the sufficient conditions for the existence of positive eigenvalues of $A$.

Lemma 2: There exists a positive eigenvalue of $A$ if any of the following two conditions hold:

(i) $\quad \lambda>\frac{\left(1-p_{11}\right)+p_{12} p_{21}}{\pi_{1}+\pi_{2} p_{21}}$

(ii) $\lambda=1,1-p_{11} \leq \min \left\{\pi_{1}, \pi_{2}\right\}$, and $p_{13} \neq 0$

Proof: The proofs of lemmas and propositions presented in this paper are detailed in [14].

Lemma 1 and 2 show the conditions for the existence of positive eigenvalue(s). These results are important since we will show later in Lemma 6 that the existence of positive eigenvalues results in the explosion of the population size. It is interesting that the sufficient conditions do not depend on several parameters, e.g. $\alpha, \beta, p_{12}$, and also $p_{21}$ in Lemma 2(ii).

The following lemma shows conditions for non-positive eigenvalues.

Lemma 3: If $\lambda=1$ and the following conditions hold then $A$ has only non-positive eigenvalues, which implies that the population will stop growing with probability 1 (see Lemma 5):

$$
\left.\begin{array}{l}
p_{12} \geq \max \left\{\pi_{1}, \pi_{2}\right\} \\
1-p_{11} \geq \pi_{1}
\end{array}\right\} \Rightarrow \gamma_{i} \leq 0 \quad \forall i
$$

If $\gamma_{1,2} \neq 0$ the eigenvectors of $A$ associated to the eigenvalues $\gamma_{1,2,3}$ are the followings:

$$
\begin{aligned}
& \mathbf{s}_{\mathbf{1}}=\left(\begin{array}{c}
\frac{p_{21}}{\beta} \\
\gamma_{1}-\frac{\lambda \pi_{1}+p_{11}-1}{\alpha} \\
\frac{p_{23}}{\beta}+\frac{a_{31} a_{12}-a_{11} a_{32}}{\gamma_{1}}
\end{array}\right) ; \\
& \mathbf{s}_{\mathbf{2}}=\left(\begin{array}{c}
\frac{p_{21}}{\beta} \\
\gamma_{2}-\frac{\lambda \pi_{1}+p_{11}-1}{\alpha} \\
\frac{p_{23}}{\beta}+\frac{a_{31} a_{12}-a_{11} a_{32}}{\gamma_{2}}
\end{array}\right) ; \mathbf{s}_{\mathbf{3}}=\left(\begin{array}{l}
0 \\
0 \\
1
\end{array}\right) .
\end{aligned}
$$

Since $\gamma_{1}>\gamma_{2}$, the three eigenvectors form a basis in $\mathbb{R}^{3}$ and the expected number of peers will be the following:

$$
\mathbf{E}\left[Z_{t}\right]=\exp (A t) Z_{0}=\sum_{i=1}^{3} c_{i} e^{\gamma_{i} t} \mathbf{s}_{\mathbf{i}}
$$

where $c_{i}, i=1,2,3$ are given as the solution of the equation system $Z_{0} \equiv\left(Z_{0}^{(1)}, Z_{0}^{(2)}, Z_{0}^{(3)}\right)=\sum_{i=1}^{3} c_{i} \mathbf{s}_{\mathbf{i}} . Z_{0}$ is the initial state of the system.

If $\gamma_{1}=0$ or $\gamma_{2}=0$ (only one of them can be zero) $A$ has only two eigenvectors, the previous calculation is not valid in this case. However, it can be shown that the rank of $A^{k}$ is 1 if $k \geq 2$, i.e.,

$$
A^{k}=\left(\mathbf{x y}^{T}\right)^{k}=\mathbf{x}\left(\mathbf{y}^{T} \mathbf{x}\right)^{k-1} \mathbf{y}^{T}
$$

where

$$
\mathbf{x}=\left(\begin{array}{c}
\frac{p_{21}}{\beta} \\
-\frac{1}{\beta} \\
\frac{a_{31} a_{12}+a_{32} a_{22}}{a_{11}+a_{22}}
\end{array}\right) \quad \mathbf{y}=\left(\begin{array}{c}
\frac{\beta}{p_{21}} \frac{\lambda \pi_{1}+p_{11}-1}{\alpha} \\
1 \\
0
\end{array}\right)
$$


and $\mathbf{y}^{T}$ is the transpose of $\mathbf{y}$. Since the third eigenvalue of $\mathrm{A}$ is $\mathbf{y}^{T} \mathbf{x}=a_{11}+a_{22}$, clearly it is not zero.

$$
\exp (A t)=I+t \underbrace{\left(A-\mathbf{x y}^{T}\right)}_{L}+\mathbf{x y}^{T} \frac{\exp \left(\mathbf{y}^{T} \mathbf{x} t\right)-1}{\mathbf{y}^{T} \mathbf{x}}
$$

where

$$
L=\frac{a_{11} a_{32}-a_{31} a_{12}}{a_{11}+a_{22}}\left(\begin{array}{lll}
0 & 0 & 0 \\
0 & 0 & 0 \\
-\frac{a_{22}}{a_{12}} & 1 & 0
\end{array}\right)
$$

It is easy to see that $L$ exerts an influence only on the number of dead peers.

Summarizing the results:

Proposition 1: The expected value of file population at time $t$ is given by

$$
\begin{gathered}
\mathbf{E}\left[Z_{t}\right]=\exp (A t) Z_{0}= \\
= \begin{cases}\sum_{i=1}^{3} c_{i} e^{\gamma_{i} t} \mathbf{s}_{\mathbf{i}} & \text { if } \gamma_{1}, \gamma_{2} \neq 0 \\
Z_{0}+t\left(L Z_{0}\right)+\mathbf{x}\left(\mathbf{y}^{T} Z_{0}\right) \frac{1}{\gamma_{2}}\left(e^{\gamma_{2} t}-1\right) & \text { if } \gamma_{1}=0 \\
Z_{0}+t\left(L Z_{0}\right)+\mathbf{x}\left(\mathbf{y}^{T} Z_{0}\right) \frac{1}{\gamma_{1}}\left(e^{\gamma_{1} t}-1\right) & \text { if } \gamma_{2}=0\end{cases}
\end{gathered}
$$

Note that $\mathbf{x}=\mathbf{s}_{\mathbf{1}}$ if $\gamma_{2}=0$ and $\mathbf{x}=\mathbf{s}_{\mathbf{2}}$ if $\gamma_{1}=0$.

This yields some important results:

Lemma 4: If $\gamma_{1}=0$ the expected numbers of active and passive offsprings are bounded while the expected value of dead offsprings grows linearly as $t$ tends to infinity.

Lemma 5: If $\gamma_{1}<0$, the process will stop growing with probability 1.

Lemma 5 shows that in case of the existence of non-positive eigenvalues the population will become extinct. It is also interesting that the sufficient conditions in Lemma 3 do not depend on $\alpha, \beta$, and $p_{21}$.

Lemma 6: If the matrix $A$ has a positive eigenvalue, i.e. $\gamma_{1}>0$, the mean number of active, passive and also dead peers tends to infinity. However, the process can still die out in this case, even with a very small probability.

This lemma shows that the existence of the positive eigenvalue which determined by the parameters as shown by Lemma 1 and 2 yields to the explosion of population size of shared files.

Proposition 2: The proportion of active, passive and dead peers converges to a deterministic vector, namely

$$
\begin{gathered}
\lim _{t \rightarrow \infty} \frac{Z_{t}}{\left\|Z_{t}\right\|_{1}}= \\
=\left\{\begin{array}{lll}
\frac{\mathbf{s}_{1}}{\left\|\mathbf{s}_{1}\right\|_{1}} & \text { if } \quad \gamma_{1}>0 & \text { and } \quad \lim _{t \rightarrow \infty}\left\|Z_{t}\right\|_{1}=\infty \\
(0,0,1) & \text { if } \quad \gamma_{1} \leq 0 \quad \text { or } \quad \lim _{t \rightarrow \infty}\left\|Z_{t}\right\|_{1}<\infty
\end{array} .\right.
\end{gathered}
$$

\section{RESULT VERIFICATION AND IMPLICATIONS}

We have implemented a simulation study to verify the results presented in the previous sections. The simulation results are shown in this section. In addition, the implications of the results and some engineering guidelines are also described and discussed.

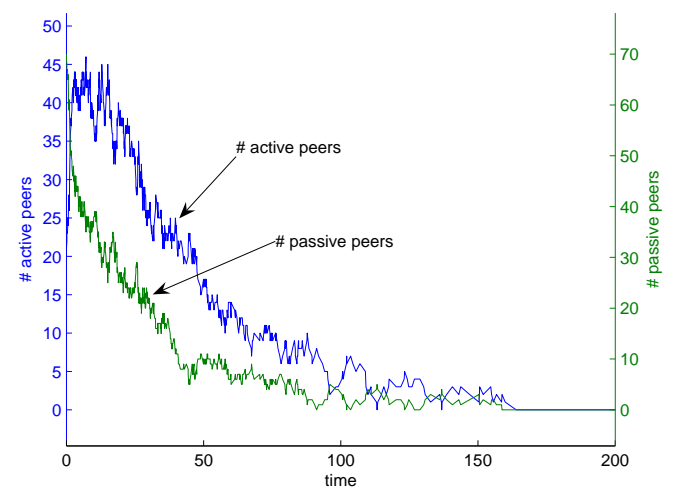

Fig. 3. The case of population extinction

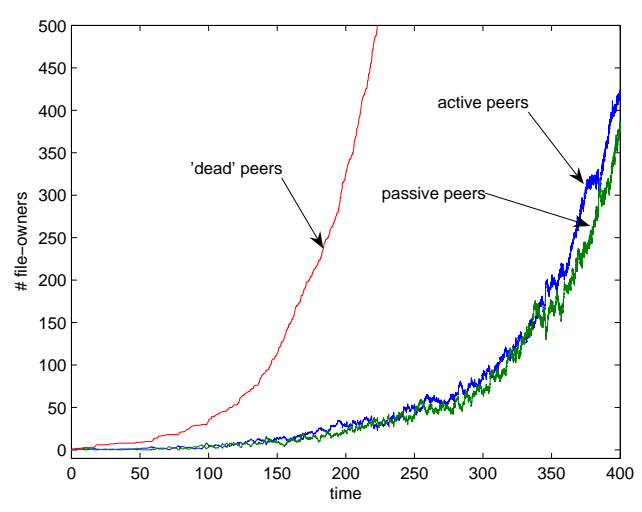

Fig. 4. The case of population explosion

\section{A. Simulation results}

The P2P system model as described in section II-B is simulated using Matlab. The parameter $\lambda$ is set to be 1, i.e., only an offspring is born from its parent at a certain point in time. We will show how the set of system parameters can predict exactly the long-term behavior of the P2P system.

Set $\alpha=4, \beta=5, \pi_{1}=\pi_{2}=0.05 ; p_{11}=0.25, p_{12}=$ $0.5, p_{21}=18 / 19$. It is easy to calculate that the conditions of Lemma 5 are satisfied. This means that the matrix $A$ has only non-positive eigenvalues, i.e. the file population dies out almost surely. The exact values of $\gamma_{1}$ and $\gamma_{2}$ are calculated to be -0.025 and -0.349 , respectively. The simulation result is shown in Fig. 3. The figure displays the change of active and passive peers in the function of time. Once the number of these peers is zero the system is extinguished. It can be seen that it happens after about 160 time units.

If we change $\pi_{1}=0.15, \pi_{2}=0.25$ while keeping the others unchanged the Lemma 2 holds. This implies that the population of all types of peers is likely to tend to infinity. The exact calculation provides $\gamma_{1}=0.015, \gamma_{2}=-0.365$. The growth of population is also justified by simulation results, see e.g. Fig. 4. Furthermore, the proportion of active, passive, and dead peers are very close to the expected values. Recall that by the result of Prop. 2 the ratio of peer types is determined by 


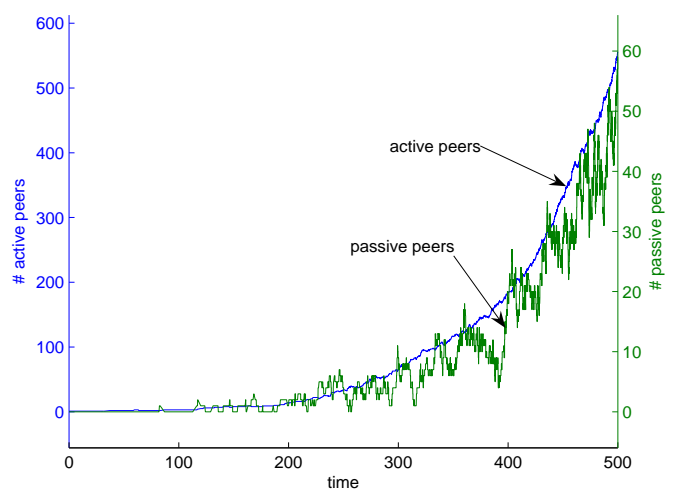

(a)

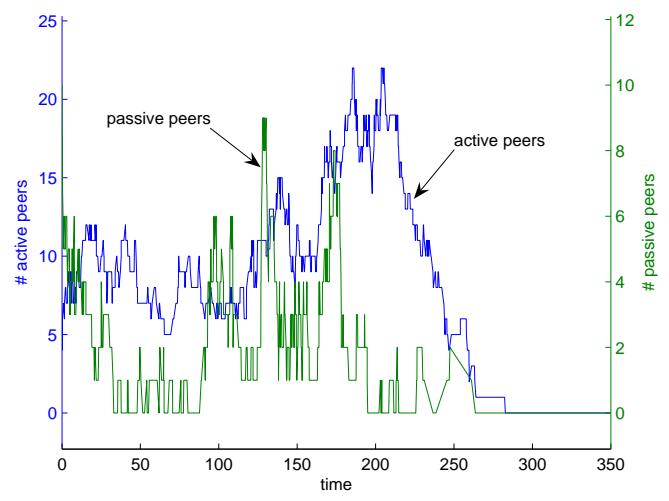

(b)

Fig. 5. The effects of free loaders

the eigenvector $\mathbf{s}_{1}$ corresponding to the maximal eigenvalue $\gamma_{1}$ which is calculated to be about $6: 5.3: 88.7$ [\%]. At the end of simulation this ratio is actually registered as $6.1: 5.4: 88.5$ [\%].

Recent results in the research of P2P systems [11], [12], [13] claim the important effects of free riding (peers that do not share). However, our analytical result shows that while free riding is an important factor in $\mathrm{P} 2 \mathrm{P}$ system performance, it is not necessarily the only one that determines the system behavior. For instance, set $\pi_{1}=0.05, \pi_{2}=0.05 ; p_{11}=$ $0.95, p_{12}=0.02, p_{21}=0.65$. In this case $90 \%$ of the downloads are free riders but with the proper setting of the other parameters the system capacity still grows, see Fig. 5(a). In contrast, in another case $\left(\pi_{1}=0.7, \pi_{2}=0.05 ; p_{11}=\right.$ $\left.0.2, p_{12}=0.1, p_{21}=0.6\right)$, Fig. $5(\mathrm{~b})$, when only $25 \%$ of the downloads is performed by freeloaders the system still collapses after a finite lifetime.

Finally, in Fig. 6 we present a case study when the system parameters are changed during the system operation. Originally the system has the parameter set $\pi_{1}=0.16, \pi_{2}=0.04$, $p_{11}=0.5, p_{12}=0.45, p_{21}=0.75$, which implies that the population will grow to infinity $\left(\gamma_{1}=0.0047\right)$. This growth can be seen in the left half of the figure. At time $t_{0} \simeq 650$ we modify some parameters such that $\pi_{1}=0.04, \pi_{2}=0.16$, while the others are unchanged. The new parameters predict

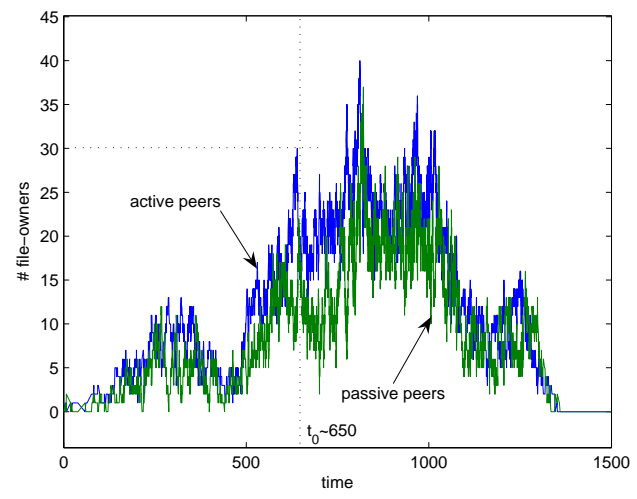

Fig. 6. The parameter set is changed at time instant $t_{0} \simeq 650$

the extinction of the population $\left(\gamma_{1}=-0.00039\right)$, which is justified by the right side of Fig. 6 . The system actually died out at about 1350 .

\section{B. Applicability of the model}

It is important to verify the model in a real P2P system. However, without the access to the source codes of these running systems the model parameters are hard to collect. Unfortunately, a normal network monitoring cannot provide all needed information to use in the model. We are working on this issue but the model verification in a working $\mathrm{P} 2 \mathrm{P}$ system is not addressed in this paper.

Nevertheless, this issue is straightforward in the case of the system operators. By putting a built-in statistical monitor in their software the model parameters can easily be estimated over time. With the help of the model the operation tendency of the system can be predicted. The impact of their possible modifications and developments can also be easily measured.

\section{Practical implications}

The proposed branching process model of $\mathrm{P} 2 \mathrm{P}$ filesharing systems provides a very clear, simple, and reliable description of the population dynamics of the shared files in the system. The model establishes several practical implications which should be carefully considered by P2P system designers and operators.

- If the population grows, the rate of growth is exponential (see (7)).

- Under some certain conditions, the long-term behavior of the system does not depend on several system parameters (see details in Lemma 2 and 5).

- As presented above we argue that the presence of free loaders is not the only factor which determines the system performance. It is one among many other important system descriptors: cooperative peers, online/offline times of peers, age times, etc.

- The model can predict exactly the long-term performance of the system using its set of parameters. A successful system design should apply rules and techniques, e.g. incentives and/or reputation index, which somehow force 
the possible ranges of system parameters such that the shared files' population grows.

- The results are also valid with different system starting conditions. The impact of new modifications, developments, or any other external circumstances, provisions can be immediately measured, estimated for an ongoing (already under operation) P2P system using a built-in statistical monitor of the software.

- In the long term with a fixed combination of parameters the system population dies out or grows exponentially or linearly (see Prop. 1). There is no other possibility. Nevertheless, in practice the system may exhibit short term stationary behavior several times during its lifetime.

\section{CONCLUSION}

In this paper we presented a mathematical model to capture the main characteristics of file-sharing peer-to-peer systems. Our model is general and flexible enough to be applied for most of the file-sharing $\mathrm{P} 2 \mathrm{P}$ applications in current use. Our results clearly predict the long-term dynamics of the population size.

We have shown that with fixed values of the parameter set the file population will either explode or die out. The important conditions that depend on the system parameters and that determine which case will happen are derived. We also derived the ratio of active, passive and dead peers in the long-term regime and showed that the growth is exponential. Using our results we have found some important practical implications: the population can explode even if most of the peers are free loaders, and the population can become extinct even if most of the peers are cooperative. We can conclude that the free loaders are not the only factor which determines the system behavior.

We proposed some useful guidelines which can help the design and control of such systems since according to our results one can control and predict the system behavior in the future.

\section{REFERENCES}

[1] K. B. Athreya and P. E. Ney, Branching Processes, 1972

[1] http://www.caida.org/analysis/workload/byapplication/sdnap/

[2] D. Qiu and R. Srikant, "Modeling and performance analysis of bittorrentlike peer-to-peer networks", ACM SIGCOMM 2004, 2004.

[3] R. Susitaival, S. Aalto and J. Virtamo, "Analyzing the dynamics and resource usage of P2P file sharing systems by a spatio-temporal model", International Workshop on P2P for High Performance Computational Sciences (P2P-HPCS'06) in conjunction with ICCS, 2006.

[4] R. Susitaival and S. Aalto, "Modelling the population dynamics and the file availability in a bitTorrent-like p2p system with decreasing peer arrival rate", IWSOS'06, 2006.

[5] X. Yang and G. de Veciana, "Service Capacity of Peer to Peer Networks", INFOCOM 2004, 2004.

[6] G. de Veciana and X. Yang, "Fairness, incentives and performance in peer-to-peer networks", Allerton Conference on Communication, Control and Computing, 2003.

[7] Y. Tian, D. Wu and K. W. Ng, "Modeling, Analysis and Improvement for BitTorrent-Like File Sharing Networks", INFOCOM 2006, 2006.

[8] L. Guo, S. Chen, Z. Xiao, E. Tan, X. Ding, and X. Zhang, "Measurement, analysis, and modeling of BitTorrent-like systems", ACM SIGCOMM Internet Measurement Conference, (IMC'05), New Orleans, LA, October 19-21, 2005.
9] S. Zhao, D. Stutzbach and R. Rejaie, "Characterizing files in the modern Gnutella network: a measurement", Proceedings of SPIE - Volume 6071 Multimedia Computing and Networking 2006, Surendar Chandra, Carsten Griwodz, Editors, 60710M, Jan. 16, 2006.

[10] Z. Ge, D. R. Figueiredo, S. Jaiswal, J. Kurose, D. Towsley, "Modeling Peer-Peer File Sharing Systems", INFOCOM 2003, 2003.

[11] E. Adar and B. Huberman, "Free riding on Gnutella", Technical report, Xerox PARC, Aug. 2000.

[12] S. Saroiu, P. K. Gummadi, S. D. Gribble, "A Measurement Study of Peer-to-Peer File Sharing Systems", in Proc. Multimedia Computing and Networking (MMCN), San Jose, January, 2002.

[13] Z. Ge, D. R. Figueiredo, S. Jaiswal, J. Kurose, D. Towsley, "Modeling Peer-Peer File Sharing Systems", in Proc Infocom, 2003.

[14] T. D. Dang, R. Pereczes, S. Molnár, "The Population Dynamics Of FileSharing Peer-to-Peer Networks", Technical Report, Budapest University of Technology and Economics, 2006. 